\title{
1. Introduction to managing AI wisely
}

\subsection{PREFACE}

Artificial intelligence (AI) refers to a field in computer science concerned with creating systems that can accomplish tasks that normally require human intelligence (Nilsson, 1971; Pesapane et al., 2018). AI systems use machine learning (self-learning) algorithms, which can learn using large amounts of data. As a result, an AI system can independently come up with, for example, new suggestions, decisions or predictions.

Our interest in the influence of AI systems on work and organizations dates back to the time when organizations hardly talked about AI, and the systems that were implemented were not yet self-learning. During that time, between 2012 and 2014, one of the then PhD students of our research group studied the implementation of data analytics in the sales process of a large telecom organization. The organization had enthusiastically switched to so-called customer lifecycle management (CLM), in short, determining performance by measuring customer statistics. This data analytics initiative would provide a new and more efficient way of working in the sales department where, at that time, account managers were still responsible for customer relations. However, what actually happened was of a completely different order. It soon became apparent that the developers of the analytics system had a different idea of what work at the sales department entailed compared to the account managers themselves. For example, the developers were convinced that their model would help account managers to approach 'the right customer, at the right time, with the right offer' out of 300 customers per manager. The account managers, on the other hand, were convinced that 'such a data-based model' could never reflect the information they obtained by 'actually talking to the customer'. The account managers had built up relational knowledge (based on the relationship with customers); for example, they memorized the names of the customer's children, which the CLM system did not record.

Nevertheless, the organization continued to implement CLM and the account managers were asked to register each time they used a CLM suggestion to make their customer contact. In practice, the account managers hardly ever used CLM, but they felt that this was expected of them by management. To meet leadership expectations, the account managers deliberately indicated 
after each customer contact that this was based on a CLM suggestion. Although almost all customer contacts remained based on the relational knowledge of the account managers, the 'fake' registrations made it seem that CLM was almost always right. This ultimately backfired for the account managers, for management decided that half of the account managers could be fired. After all, the CLM system was almost always right, which indicated that a lot less time needed to be spent building and maintaining customer relationships. When half of the account managers left the sales department, chaos ensued. Only after their departure did it become obvious that the important relational knowledge had been lost. In the end, it emerged that CLM was by no means such a 'holy grail' as was initially thought, but by then it was too late (Pachidi et al., 2021).

This was 2014, and CLM was but a relatively simple analytics system. Although some would argue that they were already dealing with an 'intelligent algorithm' (IA), it was by no means an 'intelligent system' like self-learning algorithms. This example shows, however, the potential dangers and implications that increasingly advanced, data-driven technologies can have for work and expertise. In the meantime, technological developments have not stood still, and increasingly complex self-learning algorithms are being implemented in organizations. Organizations have become increasingly confident in the possibilities of AI systems, and more and more organizations are now truly entering the field of AI.

The technological developments of recent years have meant that in our work we increasingly have to deal with AI systems that, by learning from large amounts of data, can perform tasks that were previously the purview of people. For example, they can provide legal support by digging through lengthy documents in seconds (Zhang et al., 2020) and pinpointing where specific information can be found, and they can help doctors to detect tumours that are sometimes invisible to the human eye (Kim et al., 2021). Even now, it is assumed that no technology since the start of the digital revolution some 60 years ago will have so many consequences for work and organizations. The unparalleled growth in data and technological knowledge assures that these consequences will likely only increase in the coming years.

In this book, we describe the opportunities and possibilities that the implementation of AI systems offers to organizations, while also considering the associated risks. The above example shows the danger of losing valuable knowledge based on an unsubstantiated belief in a new technology. This risk rises with the implementation of increasingly advanced AI systems. In addition, AI systems are unique because they simulate knowledge work. As an important characteristic of knowledge work is that it depends on collaboration between experts, when an AI system is implemented in a specific part of the organization, it is likely to have unexpected consequences beyond its intended 
effect. This can lead to negative results for organizations if such 'ripple effects' are not taken into account (Baptista et al., 2020). Another important characteristic of AI systems is the self-learning characteristic which makes the systems difficult to understand (also called 'black-boxed'). Fewer and fewer people are able to explain what goes on within an AI system, or the logic that suggestions or predictions are based on. This can have a major influence on who has a say in the implementation and use of AI systems.

Based on eight incumbent European organizations that introduced AI systems into their existing work processes, we describe how to deal with challenges of managing AI in practice. In our research, we encountered four main themes: organizing for data, testing and validating, algorithmic brokers and changing work. These four themes are interrelated (see Figure 1.1) and form the core chapters of this book.

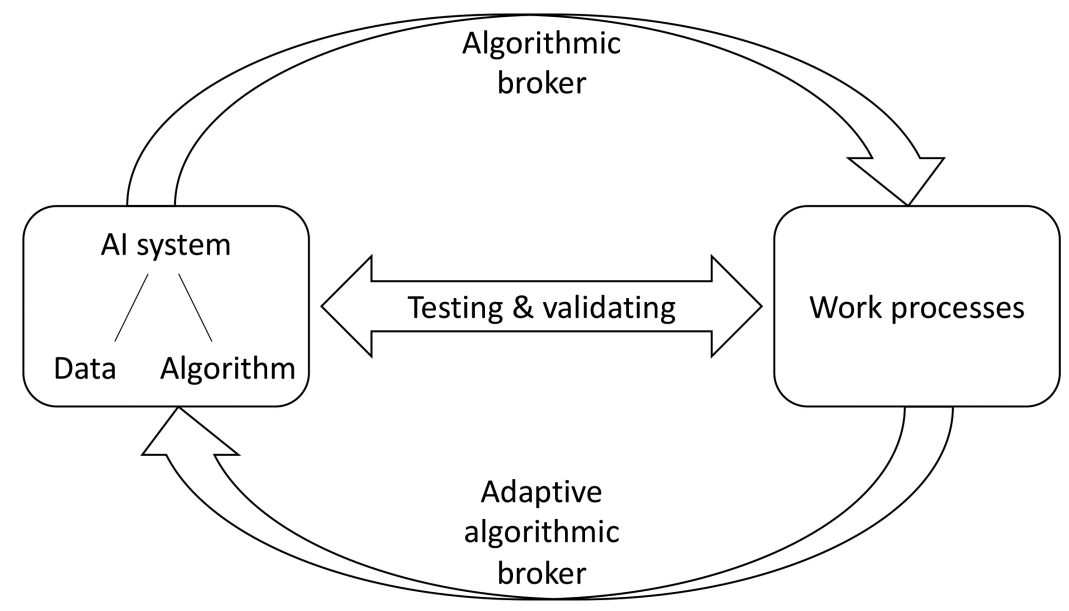

Figure 1.1 Overview of core themes

Organizing for data focuses on the management activities that are necessary to gather, construct and produce data. As the central building block of AI, data ensures that algorithms can be trained and AI systems can be developed (the left side of Figure 1.1). There is often talk of the possibilities of decontextualized Big Data for organizations. The cases described in this book show that internal, contextual data is of crucial importance for the development of AI systems that fit well with established work processes. The challenge for management is to step beyond the 'blind spot of Big Data' to organize effectively for the construction and production of data that is of added value to AI system development. 
Testing and validating refer to the need to make decisions about when and whether AI systems are 'good enough' to perform tasks (at the centre of Figure 1.1). Since AI systems can automate tasks that were previously undertaken by humans, they can potentially have major consequences for people, organizations and society. However, the black-boxed nature of AI systems makes testing and validating difficult, for it is almost impossible to find out how the results come about. The question of whether and when an AI system is good enough is therefore not only technical but also managerial. The challenge is to integrate the work processes and to test and validate against various criteria.

Algorithmic brokers refer to the fact that the outcomes of AI systems can only rarely be adopted as a 'ready-to-use product'. Instead, outcomes often have to be interpreted, filtered and translated. Here again, the black-boxed nature of AI systems makes interpreting and translating the results extremely challenging. More and more organizations are choosing to create a new role: the algorithmic broker (top of Figure 1.1). The challenge is to enable this new role to be adaptive, and to support both users and developers (bottom of Figure 1.1).

Changing work refers to a broader perspective on the consequences of the introduction of AI systems for work processes (the right side of Figure 1.1). Through the self-learning aspect of AI, it is able to generate new knowledge. When new knowledge is introduced within organizations, where knowledge is often implicit and scattered, there is a good chance that (unexpected) changes will result. The challenge is to manage the use of AI and the new knowledge in such a way that organizations are faced with as few surprises as possible, and that work is enriched through automation rather than made redundant.

\subsection{BACKGROUND OF THE BOOK}

Although the concept of AI has been around for almost 70 years, the attention from organizations towards intelligent systems has only in recent years increased exponentially. Of course, this is not accidental, as this relates to the widespread organizational access to the necessary computing power and the large amounts of data collected. Despite this, little is known about what managing AI looks like in practice. Gaining a thorough insight into this requires actually 'going into the field', to speak with developers, managers and users to observe first hand what is exactly happening in practice. We did this for this book, in which we were guided by three principles.

The first principle is narrowing down the term 'AI'. Although we cannot avoid it, we use the concept of AI with some caution. 'AI' is mainly used as an umbrella term indicating a field that covers a large number of technologies, methods and models. For example, with AI one can imply a simple calculation module in an Excel spreadsheet, but also a robot arm, or a forecasting system. 
As a result, the concept of AI runs the risk of being too generic. In addition, the term 'AI' evokes associations with magic, mysticism and even hype, that do not contribute to understanding or taking responsibility for intelligent systems. In order to make proper use of the term 'AI', in Chapter 2 we pay attention to what we mean by AI, from a technical point of view, whereby the self-learning element of algorithms plays a central role. In addition, throughout the book we speak about 'AI systems' as the technology and 'AI' as the field in which such systems reside.

The second principle is focusing on AI systems in practice. There are different schools of thought in AI research. For example, the computer science movement mainly focuses on creating and improving algorithms and is concerned with technological optimization. Another school is concerned with the ethical issues surrounding the development of AI. However, both trends focus on the stand-alone intelligent systems. Although these streams of research hold a rich body of critical and inspiring views for both managers and scientists, the disadvantage is that such studies are hardly based on concrete examples from practice. It remains unclear whether such conceptual ideas will unfold as expected in practice. To a large extent, both the successes and the dangers of AI systems are yet to be proven in practice. Existing literature on technology and work teaches us that there can be a gap between the development of technology and its actual use in daily work, which can lead to unforeseen consequences. This book provides insight into the implementation and use of AI, without losing sight of the development of technology.

The third principle is attention to managing change. Much (mainstream) scientific work focuses on the development and the implementation of AI as two separate processes. This book deviates from this focus as it deals with managing AI as an organizational change process in which development, implementation and use are intertwined. To do this, we focus our attention on the work practices within organizations, which are not about individual tasks of employees, but about how different tasks and actors relate to each other. In addition, many of the initiatives surrounding AI are focused on the possibility of intelligent systems to change work and organizations as we know them today, for example by completely replacing work or automating large parts of work. This deterministic viewpoint espouses a view where AI systems will unilaterally transform the world as we know it in a path-dependent way. In this book, we abandon this idea and look at the relations between organization and technology, whereby the technology changes the organization, but the organization also has a significant influence on the (further) development of the technology.

Our approach makes the book particularly suitable for managers, for whom the book can function as a guide. We provide management insight into the need for an integrated approach to AI. We emphasize the complexity and 
Table 1.1 Overview of cases included in the book

\begin{tabular}{l|l|l|l}
\hline Organization & Industry & AI system & Stage of implementation \\
\hline ABN AMRO & Finance & Anti money laundering system & Use \\
\hline Centraal Beheer (CB) & Insurance & Helpdesk chatbot & Use \\
\hline Dutch Police Force & Government & Predictive policing & Use \\
\hline KLM & Airline & Meals-on-Board System & Use \\
\hline LUMC (radiology) & Healthcare & Predictive tumour modelling & Implementation \\
\hline MultiCo (recruitment) & HR & Predictive people analytics & Use \\
\hline Philadelphia & Healthcare & Social robotics & Implementation \\
\hline Volkswagen & Automotive & Smart powerplants & Use \\
\hline
\end{tabular}

Note:

a. A pseudonym. The name of the organization remains anonymous for privacy reasons.

challenges, but also the opportunities that such an approach offers for the optimal development and implementation of AI in the workplace. The book's practice-oriented approach helps managers to develop their own ideas and strategy, inspired by the examples described. For this reason, we have chosen only to involve organizations that have gained concrete experience with the development and implementation of AI in existing work processes. The advantage of such an approach is that we can make well-founded statements about the success factors and challenges to managing AI in practice.

\subsection{CASE SELECTION}

The case examples in this book provide as broad a picture as possible of how organizations deal with managing AI in practice. At the start of our search, the doors to many organizations remained closed to us. Often, organizations feared losing their competitive advantage through cooperation, divulging confidential information, or even suffering reputational damage. Fortunately, we found eight large European organizations willing to cooperate that met our selection criteria (see also Table 1.1). We have anonymized only one of these organizations for privacy reasons. In the following sections, we discuss the case selection criteria used and associated limitations.

\subsubsection{Selection Criteria}

For selecting our cases, we maintained six specific criteria:

1. We have chosen to focus on incumbent organizations introducing AI into their existing work processes. Therefore, we do not examine cases where $\mathrm{AI}$ is at the core of the business model; tech companies (Google, 
Tesla, bol.com, Booking.com, or Netflix) and startups are not part of our research. The aim of our book is to provide a realistic picture of what current managers and organizations are dealing with, or will have to deal with when introducing AI at work. While the development and use of AI by tech companies is an interesting topic deserving attention, it is beyond the scope of this book.

2. In order to guarantee consistency in the use of the term 'AI', we have set the condition that the system studied must use some form of machine learning in order to be referred to as an AI system. By focusing on machine learning algorithms, we exclude earlier, perhaps more embedded systems (such as expert systems). We do this with the intention to highlight the unique qualities that make AI systems so special and influential.

3. Because our aim is to provide insight into how to manage AI in practice, we have selected cases that have developed at least a first version of an AI system and are therefore in the implementation phase (see Table 1.1). Organizations in the implementation phase usually have less experience than organizations that have already started using AI systems. We had to drop a number of cases during the data collection process that were too early-stage and where the AI system was not yet sufficiently developed. Despite the often interesting initiatives, dropped organizations had gained too little experience in the actual management of AI.

4. We have specifically chosen to source real-world examples from as wide a range of industries as possible, including professional services, healthcare providers, finance and government.

5. Because we want to keep the culture within organizations as constant as possible in our description, we have focused on Western European organizations.

6. An important precondition was, of course, whether the organizations were willing to contribute to the realization of this book, by name or by pseudonym. Although we found several closed doors in our search for suitable cases, the organizations involved in this book enthusiastically cooperated in providing information, with only one organization wanting to be anonymized.

\subsubsection{Research Limitations}

This book is not about the many failed attempts at implementing AI systems (like the example of the telecom organization at the beginning of this chapter). There are plenty of organizations that believe they can gain a competitive advantage by developing an AI system based on having a lot of data, without having a clear goal for the use of these systems. We have chosen to disregard 
these failed attempts because our focus in this book is on the choices and challenges that managers face to successfully implement AI in the long term.

There is a lot of speculation about the specific benefits of implementing AI systems: more efficiency, innovation, objectivity, and so on. However, we cannot yet answer with a resounding 'yes' the question of whether AI actually offers added value in the organizations we have studied. While we would like to argue otherwise, AI is still in its infancy when it comes to implementation in incumbent organizations. Organizations that have already gone through the entire 'AI journey' and have entered the phase of full institutionalization are therefore scarce. We see, though, that AI is not a 'danger' for work. The cases in this book show that new tasks, functions and jobs are added that we could not have foreseen in advance.

Generalization is not our goal, and we emphasize that the choice of eight cases does not necessarily make the study representative of the management of AI in practice. Our aim in this book is to describe and analyse practical cases to provide insight into how a number of leading organizations deal with the implementation and management of $\mathrm{AI}$ in practice. Because organizations all approach this in their own, unique ways, we derive a number of success factors and challenges from the cases, which we trust that managers from other organizations can benefit from.

\subsection{CHAPTER OVERVIEW}

Following this introduction, we describe the background of AI in Chapter 2. We place AI within a historical context and describe how it evolved into a broad concept that covers many different technologies today. We also explain the techniques that are classified as AI and which criteria for AI we have used for this book. In Chapter 3, we discuss current research into AI in the workplace. We discuss existing theories and perspectives around AI and work, with which we lay the foundation for the practical, case-based chapters. In addition, we also discuss current developments regarding 'responsible AI'.

In Chapters 4 to 8, we include our cases. In Chapter 4, we first discuss the research methods used and then provide a general introduction for each case. This can be used as a reading guide in the following chapters. Using the cases in Chapters 5 to 8, we discuss the four main themes we identify as specific to managing AI in practice: organizing for data, testing and validating, algorithmic brokers, and changes to work. To keep the chapters clear, we select specific elements of cases to discuss in detail in each chapter (see Table 1.2 for an overview of the division of the cases in the chapters). In Chapter 9, we end the book with practical recommendations for adopting intelligent systems. These recommendations are brought together under the acronym WISE, which 
Table 1.2 Overview of cases per chapter

\begin{tabular}{|c|c|c|}
\hline Chapter & Section & Cases \\
\hline \multirow[t]{3}{*}{$\begin{array}{l}\text { Chapter } 5 \\
\text { Organizing for data }\end{array}$} & $\begin{array}{l}\text { 5.2.1 The process of data } \\
\text { construction }\end{array}$ & $\begin{array}{l}\text { MultiCo: predictive people analytics } \\
\text { Philadelphia: social robotics }\end{array}$ \\
\hline & $\begin{array}{l}5.2 .2 \text { New roles for data } \\
\text { construction }\end{array}$ & $\begin{array}{l}\text { CB: helpdesk chatbot } \\
\text { LUMC: predictive tumour modelling }\end{array}$ \\
\hline & 5.2.3 From data to algorithm & MultiCo: predictive people analytics \\
\hline \multirow[t]{3}{*}{$\begin{array}{l}\text { Chapter } 6 \\
\text { Testing and validating }\end{array}$} & $\begin{array}{l}6.2 .1 \text { Validating using } \\
\text { technical conditions and } \\
\text { guidelines }\end{array}$ & $\begin{array}{l}\text { KLM: consumption prediction } \\
\text { ABN AMRO: money laundering prediction } \\
\text { Volkswagen: smart generators }\end{array}$ \\
\hline & $\begin{array}{l}6.2 .2 \text { Work processes as } \\
\text { part of validation }\end{array}$ & $\begin{array}{l}\text { Police: predictive policing } \\
\text { Philadelphia: social robotics }\end{array}$ \\
\hline & $\begin{array}{l}\text { 6.2.3 Managing } \\
\text { expectations and trust }\end{array}$ & $\begin{array}{l}\text { KLM: consumption prediction } \\
\text { Philadelphia: social robotics }\end{array}$ \\
\hline \multirow[t]{3}{*}{$\begin{array}{l}\text { Chapter } 7 \\
\text { Algorithmic brokers }\end{array}$} & $\begin{array}{l}\text { 7.2.1 Regular algorithmic } \\
\text { brokers }\end{array}$ & $\begin{array}{l}\text { Police: predictive policing } \\
\text { ABN AMRO: money laundering prediction }\end{array}$ \\
\hline & $\begin{array}{l}\text { 7.2.2 Adaptive algorithmic } \\
\text { brokers }\end{array}$ & $\begin{array}{l}\text { LUMC: predictive tumour modelling } \\
\text { MultiCo: predictive people analytics }\end{array}$ \\
\hline & $\begin{array}{l}\text { 7.2.3 The risks of } \\
\text { algorithmic brokering }\end{array}$ & Police: predictive policing \\
\hline \multirow[t]{3}{*}{$\begin{array}{l}\text { Chapter } 8 \\
\text { Changing work }\end{array}$} & 8.2.1 Augmenting work & $\begin{array}{l}\text { CB: helpdesk chatbot } \\
\text { LUMC: predictive tumour modelling }\end{array}$ \\
\hline & $\begin{array}{l}8.2 .2 \text { Changing } \\
\text { responsibilities and control }\end{array}$ & $\begin{array}{l}\text { MultiCo: predictive people analytics } \\
\text { Police: predictive policing }\end{array}$ \\
\hline & 8.2.3 Collective change & $\begin{array}{l}\text { CB: helpdesk chatbot } \\
\text { KLM: consumption prediction }\end{array}$ \\
\hline
\end{tabular}

implies the need for work-related insights, interdisciplinary knowledge, sociotechnical change processes, and ethical awareness in managing AI systems.

\subsection{READING GUIDE}

Though one of the leading goals of this book is to offer managers tools for managing AI systems in practice, this does not mean that only managers will benefit from reading it. For developers of AI systems, this book provides insight into the importance of collaboration with other parties involved. The challenges we identify at the end of each practical chapter can help you to anticipate the potential obstacles that may lie ahead. Especially in the transition from a technically well-functioning AI system to an AI system embedded in work processes, there are many opportunities for developers, provided that the right approach is taken towards collaboration with managers and 
users. Chapters 5 and 6 are especially helpful to develop the right stakeholder strategy. In addition, Chapter 7 offers useful tools for thinking about how the outcomes of the developed system can be translated into practice more fundamentally.

For (intended) users of AI systems, this book helps you to prepare for what the system will mean for your work. AI systems are often viewed with suspicion or even fear. With this book we give you a different perspective, in particular in Chapter 8. The practical examples of Chapters 5 and 6 provide insights into what you can do and what knowledge you need, as a user, to allow AI systems to optimally contribute to your work processes, so that work does not become unnecessarily over-automated, but is instead augmented or supported. Involvement in the development of AI systems and openness to what is new are crucial. If you are interested in the technical side of AI, Chapter 7 offers you inspiration by suggesting a new role, the algorithmic broker, where you can combine your domain expertise with more technical knowledge.

For algorithmic brokers bridging domain expertise and technical knowledge, Chapter 7 is especially important. In this chapter, we give examples of the different forms that the role of algorithmic broker can take, and what the advantages and disadvantages can be. Nevertheless, we encourage you to read the rest of the book carefully. To function effectively as an algorithmic broker, an integrated understanding of managing AI systems in practice is important.

For managers, this book is a guide to best practices related to implementing AI systems in your organization. Although it is recommended that you read this book before you start developing and introducing AI in your organization, it is not too late to implement the principles of WISE management even if you have already started. Whether you are a human resources (HR) manager, a sales manager, a production manager, or any other type of manager, this book will help you to understand how to bring AI systems and existing workflows together and the critical role you play in gathering all required expertise. In this book, we will ask a lot from you both in thinking and in action. Our aim is not to deter you, but we do hope to make it clear that the implementation of intelligent systems should not be handled lightly. The key takeaways at the end of each chapter and the recommendations from Chapter 9 will help you to make this challenge concrete and to develop a long-term vision for the WISE management of AI systems in your organization.

Do none of these apply, and are you still interested in our book? Then we hope that you will enjoy reading the coming chapters and that they will bring you new insights. 


\section{REFERENCES}

Baptista, J., Stein, M.K., Klein, S., Watson-Manheim, M.B., \& Lee, J. (2020). Digital work and organisational transformation: Emergent digital/human work configurations in modern organisations. Journal of Strategic Information Systems, 29(2). DOI: 10.1016/j.jsis.2020.101618.

Kim, B., Koopmanschap, I., Mehrizi, M.H.R., Huysman, M., \& Ranschaert, E. (2021). How does the radiology community discuss the benefits and limitations of artificial intelligence for their work? A systematic discourse analysis. European Journal of Radiology, 136, 109566.

Nilsson, N. (1971). Problem-solving methods in artificial intelligence. New York: McGraw-Hill.

Pachidi, S., Berends, H., Faraj, S., \& Huysman, M. (2021). Make way for the algorithms: Symbolic actions and change in a regime of knowing. Organization Science, 32(1), 18-41.

Pesapane, F., Codari, M., \& Sardanelli, F. (2018). Artificial intelligence in medical imaging: Threat or opportunity? Radiologists again at the forefront of innovation in medicine. European Radiology Experimental, 2(1), 35-44.

Zhang, Z., Nandhakumar, J., Hummel, J., \& Waardenburg, L. (2020). Addressing the key challenges of developing machine learning AI systems for knowledge-intensive work. MIS Quarterly Executive, 19(4), 221-238. 